\title{
A combined approach using "accelerated and a sequential life testing" applied to steel components used in highway overpasses in Brazil
}

\author{
D. I. De Souza ${ }^{1,2}$, D. R. Fonseca ${ }^{1} \&$ D. Kipper $^{1}$ \\ ${ }^{1}$ Civil Engineering Department, Fluminense Federal University, Brazil \\ ${ }^{2}$ Industrial Engineering Department, North Fluminense State University, \\ Brazil
}

\begin{abstract}
In a previous paper we applied a sequential life testing approach to a low alloy-high strength steel part used in highway overpasses in Brazil. The data was collected over a period of a few years with the steel parts working under normal use conditions. In this paper we decided to use a combined approach of accelerated life testing with sequential life testing to verify if the test results obtained under accelerated conditions could be translated to the results verified under normal use conditions with a certain degree of accuracy. To accomplish this we will apply the "Maxwell Distribution Law". We elected as sampling distribution the three-parameter Weibull model. To estimate the three parameters of the Weibull underlying sampling distribution we will use a maximum likelihood approach for censored failure data. We will be assuming a linear acceleration condition. To evaluate the accuracy (significance) of the parameter values obtained under normal conditions for the underlying three-parameter Weibull model we will apply to the expected normal failure times sequential life testing using a truncation mechanism developed previously by De Souza. An example will illustrate the application of this procedure.
\end{abstract}

Keywords: three-parameter Weibull model, low allot-high strength steel parts, sequential life testing, maximum likelihood approach. 


\section{Introduction}

The sequential life testing approach with a truncation mechanism is an attractive alternative to that of predetermined, fixed sample size hypothesis testing because of the fewer observations required for its use, especially when the underlying sampling distribution is the three-parameter Weibull model. However, it happens that sometimes the amount of time available for testing could be considerably less than the expected lifetime of the component. To overcome such a problem, there is the accelerated life-testing alternative aimed at forcing components to fail by testing them at much higher-than-intended application conditions. To go from the failure rate obtained at high stress to what a product or service is likely to experience at much lower stress, under use conditions, we will need additional modeling. These models are known as acceleration models. One possible way to translate test results obtained under accelerated conditions to normal using conditions could be through the application of the "Maxwell Distribution Law." The three-parameter Weibull distribution has a shape parameter $\beta$ that specifies the shape of the distribution, a scale parameter $\theta$ that represents the characteristic life of the distribution and a location or minimum life parameter $\varphi$, representing the minimum life of the component or part being analyzed. Its density function is given by:

$$
\mathrm{f}(\mathrm{t})=\frac{\beta}{\theta}\left(\frac{\mathrm{t}-\varphi}{\theta}\right)^{\beta-1} \exp \left[-\left(\frac{\mathrm{t}-\varphi}{\theta}\right)^{\beta}\right] ; \quad \mathrm{t} \geq 0 ; \theta, \beta, \varphi>0
$$

\section{The acceleration condition}

The "Maxwell Distribution Law" is given by the following equation:

$$
\mathrm{M}_{\mathrm{TE}}=\mathrm{M}_{\text {tot }} \times \mathrm{e}^{-\mathrm{E} / \mathrm{KT}}
$$

This law expresses the distribution of kinetic energies of molecules. In eqn (2), $\mathrm{M}_{\mathrm{TE}}$ represents the number of molecules at a particular absolute Kelvin temperature $\mathrm{T}$ (Kelvin $=273.16$ plus the temperature in Centigrade), that passes a kinetic energy greater than $\mathrm{E}$ among the total number of molecules present, $\mathrm{M}_{\mathrm{tot}}$; $\mathrm{E}$ is the energy of activation of the reaction and $\mathrm{K}$ represents the gas constant (1.986 calories per mole). Eqn (2) expresses the probability of a molecule having energy in excess of E. The development of the equations associated with the "Maxwell Distribution Law" can be found in De Souza [2\}. These equations are:

Acceleration factor $\mathrm{AF}_{2 / 1}$ at two different stress temperatures, $\mathrm{T}_{2}$ and $\mathrm{T}_{1}$ :

$$
\mathrm{AF}_{2 / 1}=\frac{\mathrm{M}_{\mathrm{TE}}(2)}{\mathrm{M}_{\mathrm{TE}}(1)}=\frac{\mathrm{e}^{-\mathrm{E} / \mathrm{KT}_{2}}}{\mathrm{e}^{-\mathrm{E} / \mathrm{KT}_{1}}}=\exp \left[\frac{\mathrm{E}}{\mathrm{K}}\left(\frac{1}{\mathrm{~T}_{1}}-\frac{1}{\mathrm{~T}_{2}}\right)\right]
$$


Term E/K:

$$
\frac{E}{K}=\frac{\ln \left(\mathrm{AF}_{2 / 1}\right)}{\left(\frac{1}{T_{1}}-\frac{1}{T_{2}}\right)}
$$

Acceleration factor $\mathrm{AF}_{2 / \mathrm{n}}$ to be applied at the normal stress temperature:

$$
\mathrm{AF}_{2 / \mathrm{n}}=\exp \left[\frac{\mathrm{E}}{\mathrm{K}}\left(\frac{1}{\mathrm{~T}_{\mathrm{n}}}-\frac{1}{\mathrm{~T}_{2}}\right)\right]
$$

Acceleration factor $\mathrm{AF}_{\theta}$ for the scale parameter $\theta$ :

$$
\mathrm{AF}_{\theta}=\frac{\theta_{\mathrm{n}}}{\theta_{\mathrm{a}}}
$$

Acceleration factor $\mathrm{AF}_{\varphi}$ for the minimum life $\varphi$ :

$$
\mathrm{AF}_{\varphi}=\frac{\varphi_{\mathrm{n}}}{\varphi_{\mathrm{a}}}
$$

According to De Souza [3], for the three-parameter Weibull model the cumulative distribution function at normal testing condition $F_{n}\left(t_{n}-\varphi_{n}\right)$ for a certain testing time $t=t_{n}$, will be given by:

$$
F_{n}\left(t_{n}-\varphi_{n}\right)=F_{a}\left(\frac{t_{n}-\frac{\varphi_{n}}{A F}}{A F}\right)=1-\exp \left[-\left(\frac{t_{n}-\frac{\varphi_{n}}{A F}}{\theta_{a} A F}\right)^{\beta_{a}}\right]
$$

Eqn 8 tells us that, under a linear acceleration assumption, if the life distribution at one stress level is Weibull, the life distribution at any other stress level is also a Weibull model. The shape parameter remains the same while the accelerated scale parameter and the accelerated minimum life are multiplied by the acceleration factor. The equal shape parameter is a necessary mathematical consequence to the other two assumptions; assuming a linear acceleration model and a Weibull sampling distribution. If different stress levels yield data with very different shape parameters, then either the Weibull sampling distribution is the wrong model for the data or we do not have a linear acceleration condition.

\section{Hypothesis tenting situations}

The hypothesis testing situations were given by De Souza [2]. For the threeparameter Weibull model we will have:

1. For the scale parameter $\theta: H_{0}: \theta \geq \theta_{0} ; \quad H_{1}: \theta<\theta_{0}$

The probability of accepting $\mathrm{H}_{0}$ will be set at (1- $\left.\alpha\right)$ if $\theta=\theta_{0}$. If $\theta=\theta_{1}$ where $\theta_{1}<\theta_{0}$, the probability of accepting $\mathrm{H}_{0}$ will be set at a low level $\gamma$. 
2. For the shape parameter $\beta: H_{0}: \beta \geq \beta_{0} ; \quad H_{1}: \beta<\beta_{0}$

The probability of accepting $\mathrm{H}_{0}$ will be set at (1- $\left.\alpha\right)$ if $\beta=\beta_{0}$. If $\beta=\beta_{1}$ where $\beta_{1}<\beta_{0}$, the probability of accepting $\mathrm{H}_{0}$ will also be set at a low level $\gamma$.

3. For the minimum life parameter $\varphi: \mathrm{H}_{0}: \varphi \geq \varphi_{0} ; \quad \mathrm{H}_{1}: \varphi<\varphi_{0}$

Again, the probability of accepting $\mathrm{H}_{0}$ will be set at $(1-\alpha)$ if $\varphi=\varphi_{0}$. Now, if we have $\varphi=\varphi_{1}$ where $\varphi<\varphi_{0}$, then the probability of accepting $\mathrm{H}_{0}$ will be once more set at a low level $\gamma$.

\section{Sequential testing}

The development of a sequential testing for the three-parameter Weibull model can be found in De Souza [3]. These equations are:

$$
\begin{gathered}
\mathrm{nln}\left(\frac{\beta_{1}}{\beta_{1}} \times \frac{\theta_{0}^{\beta_{0}}}{\beta_{0}}\right)-\ln \left[\frac{(1-\gamma)}{\alpha}\right]<\mathrm{X}_{\mathrm{i}}<\mathrm{n} \ln \left(\frac{\beta_{1}}{\beta_{1}} \times \frac{\theta_{0}^{\beta_{0}}}{\beta_{0}}\right)+\ln \left[\frac{(1-\alpha)}{\gamma}\right] \text { (9) } \\
\mathrm{X}_{\mathrm{i}}=\sum_{\mathrm{i}=1}^{\mathrm{n}}\left(\frac{\left(\mathrm{t}_{\mathrm{i}}-\varphi_{1}\right)^{\beta_{1}}}{\theta_{1}^{\beta_{1}}}-\frac{\left(\mathrm{t}_{\mathrm{i}}-\varphi_{0}\right)^{\beta_{0}}}{\theta_{0}^{\beta_{0}}}\right)-\left(\beta_{1}-1\right) \times \sum_{\mathrm{i}=1}^{\mathrm{n}} \ln \left(\mathrm{t}_{\mathrm{i}}-\varphi_{1}\right), \\
+\left(\beta_{0}-1\right) \sum_{\mathrm{i}=1}^{\mathrm{n}} \ln \left(\mathrm{t}_{\mathrm{i}}-\varphi_{0}\right)
\end{gathered}
$$

\section{Expected sample size of a sequential testing - truncation point}

The development of the equations for the expected sample size of a sequential testing for truncation purpose of a three-parameter Weibull sampling distribution can be found in De Souza [3] and [4]. These equations are:

$$
\begin{array}{r}
\mathrm{E}(\mathrm{n})=\frac{\mathrm{P}(\theta, \beta) \ln \mathrm{A}+[1-\mathrm{P}(\theta, \beta)] \ln \mathrm{B}}{\mathrm{E}(\mathrm{w})} ; \mathrm{A}=\frac{\gamma}{(1-\alpha)} ; \mathrm{B}=\frac{(1-\gamma)}{\alpha} \\
\mathrm{E}(\mathrm{w})=\ln \left(\frac{\beta_{1}}{\beta_{1}} \times \frac{\theta_{0}^{\beta_{0}}}{\beta_{0}}\right)+\left(\beta_{1}-1\right) \mathrm{E}\left[\ln \left(\mathrm{t}-\varphi_{1}\right)\right]-\left(\beta_{0}-1\right) \\
\times \mathrm{E}\left[\ln \left(\mathrm{t}-\varphi_{0}\right)\right]-\frac{1}{\beta_{1}} \mathrm{E}\left[\left(\mathrm{t}-\varphi_{1}\right)^{\beta_{1}}\right]+\frac{1}{\beta_{0}^{\beta_{0}}} \mathrm{E}\left[\left(\mathrm{t}-\varphi_{0}\right)^{\beta_{0}}\right] .
\end{array}
$$




\section{Maximum likelihood estimation for the Weibull model for censored type II data (failure censored)}

The development of the equations for the maximum likelihood estimator for the shape, scale and minimum life parameters of a Weibull sampling distribution for censored Type II data (failure censored) can be found in De Souza [3] and [4]. These equations are:

$$
\begin{gathered}
-\frac{\frac{r}{\beta}+\sum_{i=1}^{r} \ln \left(t_{i}-\varphi\right),}{\left.\sum_{i=1}^{r}\left(t_{i}-\varphi\right)^{\beta} \ln \left(t_{i}-\varphi\right)+(n-r)\left(t_{r}-\varphi\right)^{\beta} \ln \left(t_{r}-\varphi\right)\right]}=0 \\
\left.-\left[\frac{\sum_{i=1}^{r}\left(t_{i}-\varphi\right)^{\beta}+(n-r)\left(t_{r}-\varphi\right)^{\beta}}{r}-\varphi\right)^{\beta}+(n-r)\left(t_{r}-\varphi\right)^{\beta}\right] \\
+\beta \times\left[\sum_{i=1}^{r}\left(t_{i}-\varphi\right)^{\beta-1}+(n-r)\left(t_{r}-\varphi\right)^{\beta-1} \sum_{i=1}^{r} \frac{1}{\left(t_{i}-\varphi\right)}\right. \\
\theta=\left[\sum_{i=1}^{r}\left(t_{i}-\varphi\right)^{\beta}+(n-r)\left(t_{r}-\varphi\right)^{\beta} / r\right)^{1 / \beta}
\end{gathered}
$$

Eqns (13) and (14) must be solved iteratively. The existence of solutions to the above set of eqns (13) and (14) has been frequently addressed by researchers as there can be more than one solution or none at all; see Zanakis and Kyparisis [5].

The standard maximum likelihood method for estimating the parameters of the three-parameter Weibull model can have problems since the regularity conditions are not met, see Zanakis and Kyparisis [5], Murthy, et al. [6] and Blischke [7]. To overcome this regularity problem, one of the approaches proposed by Cohen et al. [8] is to replace eqn (14) with the equation

$$
\mathrm{E}(\varphi)=\varphi_{\mathrm{n}}=\mathrm{t}_{1}-\frac{\theta_{\mathrm{n}}}{\mathrm{n}^{1 / \beta}} \Gamma\left(\frac{1}{\beta}+1\right)
$$

Here, $t_{1}$ is the first order statistic in a sample of size $\underline{n}$. The complete solution of eqn (16) can be found in De Souza [3] and [4]. 
Table 1: Failure times (cycles) of steel parts tested under accelerated temperature conditions $(480 \mathrm{~K})$.

\begin{tabular}{|c|c|c|}
\hline 625,536 & 772,353 & 815,092 \\
\hline 876,137 & 922,869 & 991,378 \\
\hline $1,050,475$ & $1,117,513$ & $1,254,973$ \\
\hline
\end{tabular}

Table 2: $\quad$ Failure times (cycles) of steel parts tested under accelerated temperature conditions $(520 \mathrm{~K})$.

\begin{tabular}{|l|l|l|}
\hline 532,371 & 657,322 & 693,695 \\
\hline 745,648 & 785,421 & 843,726 \\
\hline 894,021 & 951,075 & $1,068,062$ \\
\hline
\end{tabular}

\section{Example}

We are trying to determine the values of the shape, scale and minimum life parameters of an underlying three-parameter Weibull model, representing the life cycle of a new low alloy-high strength steel part. Once a life curve for this steel part is determined, we will be able to verify using sequential life testing, if new units produced will have the necessary required characteristics. It happens that the amount of time available for testing is considerably less than the expected lifetime of the component. So, we will have to rely on an accelerated life testing procedure to obtain failure times used on the parameters estimation procedure. The steel part has a normal operating temperature of $296 \mathrm{~K}$ (about 23 degrees Centigrade). Under stress testing at $480 \mathrm{~K}, 12$ steel parts were subjected to testing, with the testing being truncated at the moment of occurrence of the ninth failure. Table 1 shows these failure time data (cycles).

Now, under stress testing at $520 \mathrm{~K}, 12$ steel parts were again subjected to testing, with the testing being truncated at the moment of occurrence of the ninth failure. Table 2 shows these failure time data (cycles).

Using the maximum likelihood estimator approach for the shape parameter $\beta$; for the scale parameter $\theta$ and for the minimum life $\varphi$ of the Weibull model for censored Type II data (failure censored), we obtain the following values for these three parameters under accelerated conditions of testing:

$$
\begin{aligned}
& \text { At } 480 \text { K. } \beta_{1}=\beta_{n}=\beta=3.28 ; \theta_{1}=997,891 \text { cycles; } \varphi_{1}=183,099 \text { cycles } \\
& \text { At } 520 \text { K. } \beta_{2}=\beta_{n}=\beta=3.21 ; \theta_{2}=849,269 \text { cycles; } \varphi_{2}=155,259 \text { cycles }
\end{aligned}
$$

The shape parameter did not change with $\beta \approx 3.25$. The acceleration factor for the scale parameter $\mathrm{AF} \theta_{2 / 1}$ will be given by:

$$
\mathrm{AF} \theta_{2 / 1}=\theta_{1} / \theta_{2}=997,891 / 849,269
$$


Using eqn (4), we can estimate the term $\mathrm{E} / \mathrm{K}$ :

$$
\frac{\mathrm{E}}{\mathrm{K}}=\frac{\ln \left(\mathrm{AF}_{2} / 1\right)}{\left(\frac{1}{\mathrm{~T}_{1}}-\frac{1}{\mathrm{~T}_{2}}\right)}=\frac{\ln (997,891 / 849,269)}{\left(\frac{1}{480}-\frac{1}{520}\right)}=1,006.3
$$

Using now eqn (5), the acceleration factor for the scale parameter to be applied at the normal stress temperature $\mathrm{AF}_{2 / \mathrm{n}}$, will be:

$$
\mathrm{AF}_{2 / \mathrm{n}}=\exp \left[\frac{\mathrm{E}}{\mathrm{K}}\left(\frac{1}{\mathrm{~T}_{\mathrm{n}}}-\frac{1}{\mathrm{~T}_{2}}\right)\right]=\exp \left[1,006.3\left(\frac{1}{296}-\frac{1}{520}\right)\right]=4.32
$$

Therefore, the scale parameter of the steel part at normal operating temperatures is estimated to be:

$$
\theta_{\mathrm{n}}=\mathrm{AF}_{2} / \mathrm{n} \times \theta_{2}=4.32 \times 849,269=3,668,842 \text { cycles }
$$

The acceleration factor for the minimum life parameter $\mathrm{AF} \varphi_{2 / 1}$ will be given by:

$$
\operatorname{AF} \varphi_{2 / 1}=\frac{\varphi_{1}}{\varphi_{2}}=\frac{183,099}{155,259}
$$

Again applying eqn (4), we can again estimate the term E/K. Then:

$$
\frac{\mathrm{E}}{\mathrm{K}}=\frac{\ln \left(\mathrm{AF}_{2 / 1}\right)}{\left(\frac{1}{\mathrm{~T}_{1}}-\frac{1}{\mathrm{~T}_{2}}\right)}=\frac{\ln (183,099 / 155,259)}{\left(\frac{1}{480}-\frac{1}{520}\right)}=1,029.2
$$

Using once more eqn (5), the acceleration factor for the minimum life parameter, to be applied at the normal stress temperature $\mathrm{AF} \varphi_{2 / \mathrm{n}}$, will be:

$$
\mathrm{AF}_{2 / \mathrm{n}}=\exp \left[\frac{\mathrm{E}}{\mathrm{K}}\left(\frac{1}{\mathrm{~T}_{\mathrm{n}}}-\frac{1}{\mathrm{~T}_{2}}\right)\right]=\exp \left[1,029.2\left(\frac{1}{296}-\frac{1}{520}\right)\right]=4.47
$$

Then, as we expected, $\mathrm{AF}_{\theta}=4.32 \approx \mathrm{AF}_{\varphi}=4.47 \approx \mathrm{AF}=4.4$. Finally, the minimum life parameter of the component at normal operating temperature is estimated to be:

$$
\varphi_{\mathrm{n}}=\mathrm{AF} \varphi_{2 / \mathrm{n}} \times \varphi_{2}=4.4 \times 155,259=683,140 \text { cycles }
$$

Then, the steel part life when operating at normal use conditions could be represented by a three-parameter Weibull model having a shape parameter $\beta$ of 3.25 ; a scale parameter $\theta$ of $3,668,842$ cycles and a minimum life $\varphi$ of 683,140 cycles. To evaluate the accuracy (significance) of the three-parameter values obtained under normal conditions for the underlying Weibull model we will apply, to the expected normal failure times, a sequential life testing using a 
truncation mechanism developed by De Souza [3] and [4]. These expected normal failure times will be acquired by multiplying the nine failure times obtained under accelerated testing conditions at $520 \mathrm{~K}$ given by Table 2 by the accelerating factor AF of 4.4. It was decided that the value of $\alpha$ was 0.05 and $\gamma$ was 0.10. In this example, the following values for the alternative and null parameters were chosen: alternative scale parameter $\theta_{1}=3,700,000$ cycles, alternative shape parameter $\beta_{1}=2.9$ and alternative location parameter $\varphi_{1}=$ 700,000 cycles; null scale parameter $\theta_{0}=3,300,000$ cycles, null shape parameter $\beta_{0}=3.3$ and null minimum life parameter $\varphi_{0}=550,000$ cycles. Now electing $\mathrm{P}(\theta, \beta, \varphi)$ to be 0.01 , we can calculate the expected sample size $\mathrm{E}(\mathrm{n})$ of this sequential life testing under analysis. Using now equation (12) and eqn (11), the expression for the expected sample size of the sequential life testing for truncation purpose $\mathrm{E}(\mathrm{n})$, we will have $\mathrm{E}(\mathrm{w})=0.325$.

$$
\text { Now, with } \mathrm{P}(\theta, \beta, \varphi)=0.01 ; \ln (\mathrm{B})=\ln \left[\frac{(1-\gamma)}{\alpha}\right]=\ln \left[\frac{(1-0.10)}{0.05}\right]=2.8904 \text {, }
$$

and also with $\ln (\mathrm{A})=\ln \left(\frac{\gamma}{1-\alpha}\right)=\ln \left(\frac{0.10}{1-0.05}\right)=-2.2513$, we will have:

$$
\begin{gathered}
\mathrm{P}(\theta, \beta) \ln (\mathrm{A})+[1-\mathrm{P}(\theta, \beta)] \ln (\mathrm{B})=-0.01 \times 2.2513+0.99 \times 2.8904=2.8390 \\
\text { Finally: } \mathrm{E}(\mathrm{n})=\frac{2.8390}{0.325}=8.73 \approx 9 \text { items }
\end{gathered}
$$

So, we could make a decision about accepting or rejecting the null hypothesis $\mathrm{H}_{0}$ after the analysis of observation number 9. Using eqns (9) and (10) and the nine failure times obtained under accelerated conditions at $520 \mathrm{~K}$ given by Table 2 , multiplied by the accelerating factor $\mathrm{AF}$ of 4.4, we calculate the sequential life testing limits. Table 3 shows these expected failure time data (cycles).

Figure 1 shows the sequential life-testing for the three-parameter Weibull model.

A line is drawn through the origin of the graph parallel to the accept and reject lines. The decision to accept or reject $\mathrm{H}_{0}$ simply depends on which side of the line the final outcome lays. Obviously this procedure changes the levels of $\alpha$ and $\gamma$ of the original test; however, the change is slight if the truncation point is not too small [9]. As we can see in Figure 1, the null hypothesis $\mathrm{H}_{0}$ should be accepted since the final observation (observation number 9) lies on the side of the line related to the acceptance of $\mathrm{H}_{0}$.

Table 3: Failure times (cycles) of steel parts tested under normal temperature conditions (296 K).

\begin{tabular}{|c|c|c|}
\hline $2,342,432$ & $2,892,217$ & $3,052,258$ \\
\hline $3,280,851$ & $3,455,852$ & $3,712,394$ \\
\hline $3,933,692$ & $4,184,730$ & $4,699,473$ \\
\hline
\end{tabular}




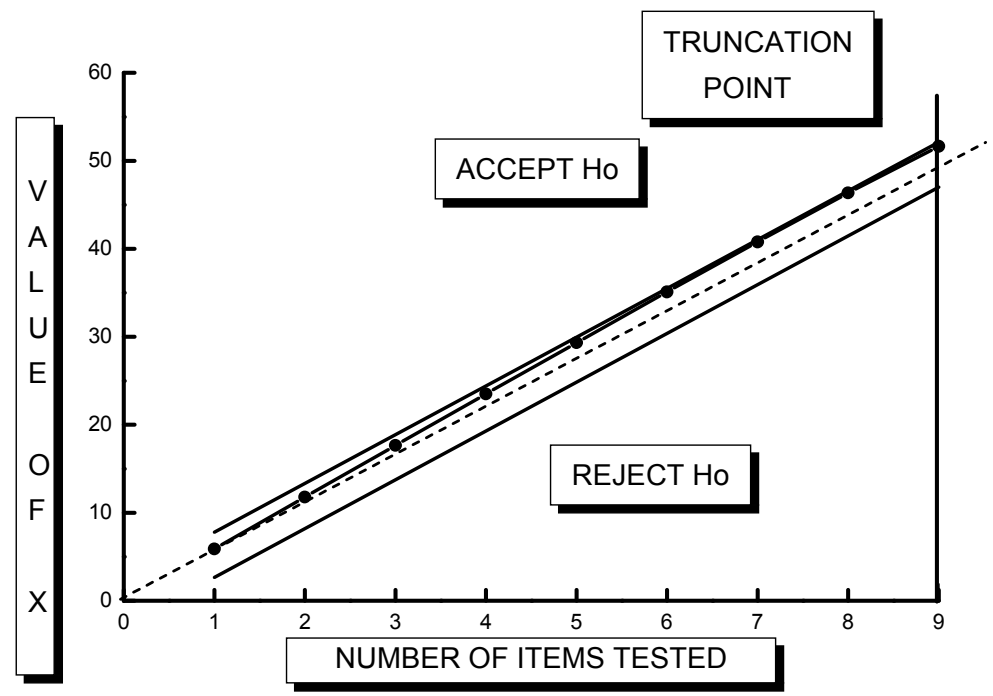

Figure 1: A truncation procedure for the sequential testing - three-parameter Weibull model.

\section{Conclusions}

In this work we life-tested a new industrial product using an accelerated mechanism. We assumed a linear acceleration condition. To estimate the parameters of the three-parameter Weibull model we used a maximum likelihood approach for censored failure data, since the life-testing will be terminated at the moment the truncation point is reached. The shape parameter remained the same while the accelerated scale parameter and the accelerated minimum life parameter were multiplied by the acceleration factor. The equal shape parameter is a necessary mathematical consequence of the other two assumptions; that is, assuming a linear acceleration model and a three-parameter Weibull sampling distribution. If different stress levels yield data with very different shape parameters, then either the three-parameter Weibull sampling distribution is the wrong model for the data or we do not have a linear acceleration condition. In order to translate test results obtained under accelerated conditions to normal using conditions we applied some reasoning given by the "Maxwell Distribution Law." To evaluate the accuracy (significance) of the three-parameter values estimated under normal conditions for the underlying Weibull model we employed, to the expected normal failure times, a sequential life testing using a truncation mechanism developed by De Souza [3, 4]. These expected normal failure times were acquired by multiplying the nine failure times obtained under accelerated testing conditions at $520 \mathrm{~K}$ given by Table II, by the accelerating 
factor AF of 4.4. Without using the truncation mechanism developed in this work, we were not able to reach a decision about accepting or rejecting a null hypothesis $\mathrm{H}_{0}$ after obtaining 9 observations. Applying the developed truncation mechanism, the decision of accepting the null hypothesis was reached with the analysis of only these 9 observations or items. This fact shows the advantage of using such a truncation mechanism in a sequential life test approach.

\section{References}

[1] De Souza, Daniel I. A Maximum Likelihood Approach Applied to a Truncated Sequential Life Testing with an Underlying Three-Parameter Weibull Model. In: Raj B. K. Rao \& David U Mba Eds. COMADEM 2005 Condition Monitoring and Diagnostic Engineering Management, University Press, 2005. v.01. p. 93-103. Cranfield, Bedfordshire, UK.

[2] De Souza, Daniel I. A Maximum Likelihood Approach Applied to an Accelerated Life Testing with an Underlying Three-Parameter Inverse Weibull Model In: Raj B. K. Rao \& David U Mba Eds. COMADEM 2005 Condition Monitoring and Diagnostic Engineering Management, University Press, 2005. v.01. p. 63-72. Cranfield, Bedfordshire, UK.

[3] De Souza, Daniel I. Sequential Life-Testing with Truncation Mechanisms for Underlying Three-Parameter Weibull and Inverse Weibull Models. In Raj B. K. Rao, B. E. Jones \& R. I. Grosvenor Eds.; COMADEM Conference, Cambridge, U.K., August 2004, p. 260-271, Comadem International, Birmingham, U.K.

[4] De Souza, Daniel I. Sequential Life Testing with a Truncation Mechanism for an Underlying Three-Parameter Weibull Model, ICHEAP-6, Chemical Engineering Transactions, Vol 3, pp. 557-562, 2003, Sauro Pierucci (ed), Pisa, Italy.

[5] Zanakis, S. H. \& Kyparisis, J. A Review of Maximum Likelihood Estimation Methods for the Three Parameter Weibull Distribution. Journal of Statistical Computation and Simulation, 25, 53-73, 1986.

[6] Murthy, D. N. P.; Xie, M. and Hang, R. 2004. Weibull Models. In Wiley Series in Probability and Statistics, John Wiley \& Sons, Inc., New Jersey.

[7] Blischke, W. R. On non-regular estimation II. Estimation of the Location Parameter of the Gamma and Weibull Distributions, Communications in Statistics, 3, 1109-1129, 1974.

[8] Cohen, A. C.; Whitten, B. J. \& Ding, Y. Modified Moment Estimation for the Three-Parameter Weibull Distribution. Journal of Quality Technology 16, 159-167. 1984.

[9] Kapur, Kailash \& Lamberson, Leonard R. 1977. Reliability in Engineering Design. New York: John Willey \& Sons, Inc. 